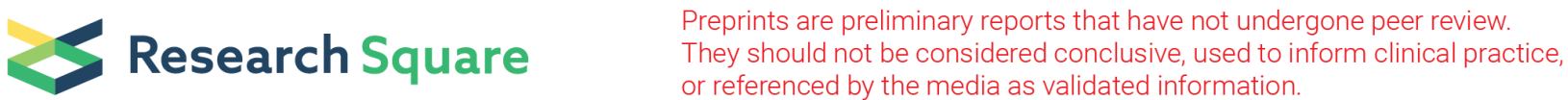

\section{Interleukin-6-knockdown of chimeric antigen receptor-modified T cells significantly reduces IL-6 release from monocytes}

\section{Liqing Kang}

Institute of Biomedical Engineering and Technology,Shanghai Engineering Research Center of Molecular Therapeutics and New Drug Development,School of Chemistry and Molecular Engineering,East China Normal University https://orcid.org/0000-0002-9840-9060

\section{Xiaowen Tang}

Institute of Blood and Marrow Transplantation $\otimes$ Collaborative Innovation Center of Hematology,Soochow University $₫$ Suzhou ,China

\section{Jian Zhang}

Institue of Blood and Marrow Transplantation,Collaborative Innovation Center of Hematology,Soochow University,Suzhou,China

\section{Minghao Li}

Institute of Biomedical Engineering and Technology,Shanghai Engineering Research Center of Molecular Therapeutics and New Drug Development,School of Chemistry and Molecular Engineering,East China Normal University,NO,3663 North Zhongshan Road,Shanghai,200065,China.

\section{Nan Xu}

Institute of Biomedical Engineering and Technology खShanghai Engineering Research Center of Molecular Therapeutic and New Drug Development\School of Chemistry and Molecular Engineering区East

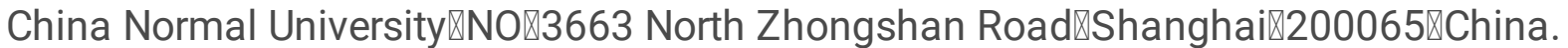

\section{Wei Qi}

Institute of Biomedical Engineering and Technology खShanghai Engineering Research Center of

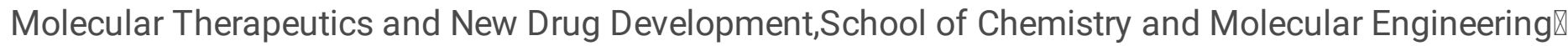
East China Normal University

\section{Jingwen Tan}

Institute of Biomedical Engineering and Technology $\llbracket$ Shanghai Engineering Research Center of

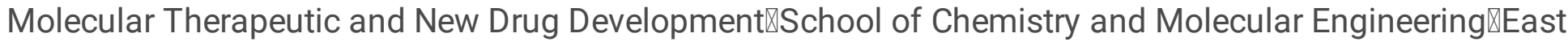

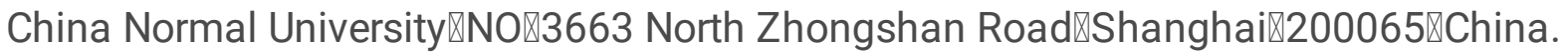

\section{Xiaoyan Lou}

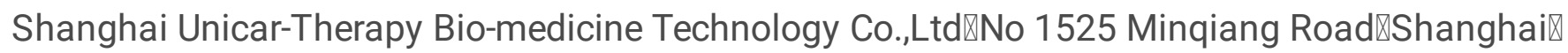
201612खChina.

\section{Zhou Yu}


Shanghai Unicar-Therapy Bio-medicine Technology Co.,Ltd,No 1525 Minqiang Road\Shanghai $201612 \rrbracket$ China.

\section{Juanjuan Sun}

Shanghai Unicar-Therapy Bio-medicine Technology Co.,Ltd,No 1525 Minqiang Road $₫$ Shanghai $201612 \rrbracket$ China.

\section{Zhenkun Wang}

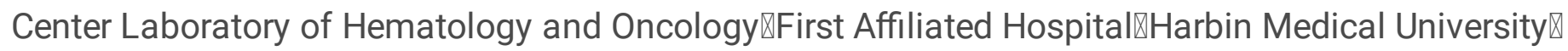
Harbin 150001هHeilongjiang Province『China.

\section{Haiping Dai}

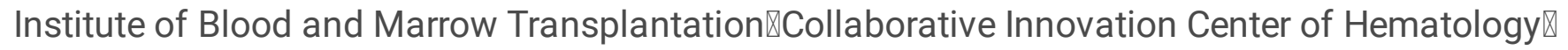

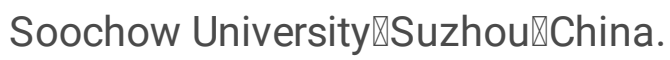

\section{Jia Chen}

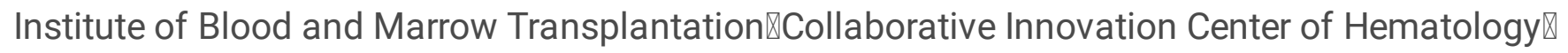

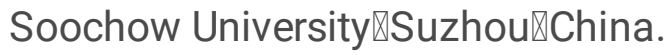

\section{Depei Wu}

National Clinical Research Center for Hematologic Diseases $₫$ Jiangsu Institute of Hematology $₫ T h e$ First Affiliated Hospital of Soochow University $₫$ Suzhou凶China.

\section{Lei Yu ( $\nabla$ ylyh188@163.com )}

https://orcid.org/0000-0002-1083-0361

\section{Research}

Keywords: Chimeric antigen receptor (CAR), T cell, interleukin-6 (IL-6), shRNA, safety

Posted Date: March 11th, 2020

DOl: https://doi.org/10.21203/rs.3.rs-16656/v1

License: (c) (1) This work is licensed under a Creative Commons Attribution 4.0 International License. Read Full License

Version of Record: A version of this preprint was published at Experimental Hematology \& Oncology on June 8th, 2020. See the published version at https://doi.org/10.1186/s40164-020-00166-2. 


\section{Abstract}

Background T cells expressing a chimeric antigen receptor (CAR) engineered to target CD19 can treat leukemia effectively but also increase the risk of complications such as cytokine release syndrome (CRS) and CAR T cell related encephalopathy (CRES) driven by interleukin-6 (IL-6). Here, we investigated whether IL-6 knockdown in CART-19 cells can reduce IL-6 secretion from monocytes, which may reduce the risk of adverse events.

Methods Supernatants from cocultures of regular CART-19 cells and B lymphoma cells were added to monocytes in vitro, and the IL- 6 levels in monocyte supernatants were measured $24 \mathrm{~h}$ later. IL-6 expression was knocked down in regular CART-19 cells by adding a short hairpin RNA (shRNA) (termed sSCART-19) expression cassette specific for IL-6 to the conventional CAR vector. Transduction efficiency and cell proliferation were measured by flow cytometry, and cytotoxicity was measured by evaluating the release of lactate dehydrogenase into the medium. Gene expression was assessed by qRT-PCR and RNA sequencing. A xenograft leukemia mouse model was established by injecting NOD/SCID/Yc-/- mice with luciferase-expressing B lymphoma cells, and then the animals were treated with regular CART-19 cells or ssCART-19. Tumor growth was assessed by bioluminescence imaging.

Results Both recombinant IL- 6 and activated regular CART-19 cells expressing IL- 6 triggered IL- 6 release by monocytes. IL- 6 knockdown in SSCART- 19 cells dramatically reduced IL- 6 release from monocytes without reducing cytotoxic activity. Mice treated with SSCART-19 cells showed lower IL-6 levels in the serum than mice treated with regular CART-19 cells, but tumor growth and survival were similar between the animal groups.

Conclusion IL- 6 released from activated CAR T cells may be one of the main initiators of the release of IL6 from monocytes that can drive CRS. IL- 6 knockdown in SSCART-19 cells reduces monocyte release of IL6 both in vitro and in vivo without affecting antitumor efficacy. The IL- 6 knockdown strategy may provide a useful and promising way to improve the safety of CAR T cell therapy.

\section{Background}

T cells expressing a chimeric antigen receptor (CAR) engineered to target CD19 are an effective treatment for refractory and relapsed acute B lymphoblastic leukemia[1]. However, CAR T cell therapy is also associated with severe life-threatening side effects[2-5], which have greatly hindered its implementation. As many as $70 \%$ of patients on this therapy experience severe cytokine release syndrome (sCRS)[4], and $40 \%$ of patients experience severe CAR T cell-related encephalopathy (sCRES) $[1,6,7]$. Both of these complications are associated with high IL-6 levels in the circulation and cerebrospinal fluid[2]. While tocilizumab and hormone therapy can be used to treat SCRS[8], these approaches are costly and increase the risk of additional side effects such as infection, and more importantly, monoclonal antibodies such as tocilizumab cannot reach damaged areas in the brain because of the brain-blood barrier[9]. Hormone therapy can also impair CAR T cell function and weaken therapeutic efficacy[10,11]. A novel and 
effective method to improve the safety of CAR T cell clinical application without affecting the efficacy of CAR T cells is urgently needed.

After adoptive transfer into patients, CAR T cells are activated by B cells to proliferate and release various proinflammatory cytokines, including interleukin (IL)-6, interferon (IFN)-y, MCP-1, and granulocyte macrophage colony-stimulating factor (GM-CSF)[12-14]. This activation leads to target cell lysis and widespread immune activation[12,15]. At the same time, the cytokines produced by the CAR T cells or other immune cells, such as monocytes, in response to CAR T cell infusion appear to drive CRS and CRES[12, 15].

Among these cytokines, IL-6 appears to be a key driver of SCRS, sCRES and related death[16]. One study reported that these syndromes are triggered by CAR T cell activation and proliferation after recognition of CD19 + target cells and are characterized by elevated serum levels of IL- 6 and IFN- [12][15]. Elevated IL- 6 expression has also been associated with neurotoxicity caused by CAR T cells[17]. While CAR T cells themselves produce IL- 6 after infusion, other cells, including monocytes, dendritic cells, B lymphocytes and endothelial cells, may contribute to IL- 6 release[3, 18-21]. High levels of cytokines such as IL- 6 and IFN- $\gamma$ may activate endothelial cells, resulting in the release of Von Willebrand Factor (VWF) and angiopoietin-2, which in turn amplify endothelial activation[22]. The mechanisms that trigger the rapid release of large amounts of IL- 6 from monocytes and endothelial cells during CAR T therapy are unclear.

Given the important role of IL- 6 in complications related to CAR T cell therapy, we first investigated whether CAR T cell-derived IL- 6 activates and triggers monocyte release of proinflammatory cytokines, including IL-6, and then examined whether IL- 6 production in monocytes can be reduced by knocking down IL- 6 gene expression in CAR T cells without affecting their antitumor efficacy. The current study will provide useful data for the development of a new strategy to improve the safety of CAR T cell therapy.

\section{Materials And Methods}

\section{Generation of IL-6 shRNA-expressing CAR constructs}

We designed 8 different short hairpin RNA (shRNA) sequences targeting the 3 ' untranslated region (UTR) of the human IL- 6 gene using human $\mathrm{U} 6$ as the promotor (sequence:

5'AATTCAAAAAAGGGCACAGAACTTATGTTGTTCTCGAGAGAACAACATAAGTTCTGTGC-3'). Different shRNAs were synthesized by GENERAY Biotech (Shanghai, China) and inserted into a CAR construct (Unicar-Therapy Bio-medicine Technology Co., Ltd., Shanghai, China) containing a CD19-targeted singlechain variable fragment (FMC63), the EF1a promoter, the costimulatory 4-1BB domain, and the CD3 zeta domain. The construct was cotransfected with three packaging plasmids into HEK 293T packaging cells, and the resulting lentiviruses were isolated, concentrated by ultracentrifugation, and immediately stored at $-80^{\circ} \mathrm{C}$.

\section{CART-19 cells and target cell lines}


Peripheral blood mononuclear cells (PBMCs) were obtained from healthy donors by gradient centrifugation using Lymphoprep ${ }^{\mathrm{TM}}$ (Oriental Hua Hui, Beijing, China). For CAR T cell preparation, $\mathrm{T}$ lymphocytes were purified using anti-CD3 positive-selection beads (Miltenyi Biotec, Bergisch-Gladbach, Germany) and stimulated with anti-CD3/CD28 monoclonal antibodies (Miltenyi Biotec) for 18-24 h. The T cells were then transduced with recombinant lentiviral vectors for $48 \mathrm{~h}$. The CAR T cells were cultured for 12-14 days in AIM-V medium (Gibco, NY, USA) supplemented with $10 \%$ autologous human serum, 100 $\mathrm{IU} / \mathrm{mL}$ recombinant human IL-2 (PeproTech, Rocky Hill, USA), $5 \mathrm{ng} / \mathrm{mL}$ recombinant human IL-7 (PeproTech) and $5 \mathrm{ng} / \mathrm{mL}$ recombinant human IL-15 (PeproTech).

Monocytes were collected as previously described[23]. In total, $2 \times 10^{6}$ cells were seeded per well in $1 \mathrm{~mL}$ of RPMI 1640 medium (Gibco) supplemented with $0.1 \mathrm{mmol} / \mathrm{L}$ minimum essential medium containing nonessential amino acids (Solarbio, Beijing, China), $2 \mathrm{mmol} / \mathrm{L}$ L-glutamine (Thermo Fisher, Waltham, USA), and $10 \%$ fetal bovine serum (FBS; Gibco). Cells were supplemented with fresh medium every two days, harvested using $2 \mathrm{mmol} / \mathrm{L}$ ethylenediaminetetraacetic acid (EDTA, Klamar, Shanghai, China) and stained with an APC-conjugated antibody against CD14 (BD Bioscience, New Jersey, USA) to confirm differentiation.

A CD19-expressing Burkitt human B lymphoma (Raji) cell line and the K562 human leukemia cell line (ATCC, Manassas, VA, USA) were used as target cells in vitro and as control cells. The cells were maintained in RPMI 1640 medium (Gibco) supplemented with 10\% FBS (HyClone, UT, USA) according to manufacturer protocols. Raji cells were transfected with a firefly luciferase construct (Unicar-Therapy Biomedicine Technology Co., Ltd., Shanghai, China) using a lentiviral vector, and stably transfected cells were selected using puromycin.

\section{Coculture assay}

An "effector/target cell" culture model was used for the coculture assay. CAR T cells (effector cells) and Raji cells (target cells) were incubated at a ratio of $5: 1$ for $24 \mathrm{~h}$ in $100 \mu \mathrm{L}$ of T cell culture medium AIM-V (Gibco) in 96-well plates. The culture supernatant was then added to primary monocytes, and IL-6 levels in the supernatant were measured using the Th1/Th2 Cytometric Bead Array Kit II (BD Bioscience). The IL- 6 levels at $0 \mathrm{~h}$ (immediately after the addition of the coculture supernatant) were used as the baseline.

Then, $10 \mathrm{ng} / \mathrm{mL}$ human recombinant IL-6, IL-2, IL-1 or IFN-y (PeproTech) was added to the monocyte cultures. Supernatant samples were collected at $6 \mathrm{~h}, 24 \mathrm{~h}$ and $48 \mathrm{~h}$ after beginning the coincubations of the different cytokines and monocytes, and monocytes incubated with an equal volume of saline were used as a control for comparisons of cytokine profiles in this experiment.

For re-stimulation cocultures, CAR T cells expressing an shRNA against IL-6 (sSCART-19) or regular CAR T cells were cocultured with Raji cells at a ratio of $5: 1$ for 6 days, and the different types of CAR T cells were 
re-stimulated with Raji cells every $36 \mathrm{~h}$. Supernatants were collected every $12 \mathrm{~h}$, and the levels of IL-6, IFNY and IL-2 were measured with the Th1/Th2 Cytometric Bead Array Kit II.

\section{Flow cytometry}

All samples were washed twice in $0.1 \mathrm{~mL}$ of phosphate-buffered saline (PBS) containing $2 \%$ FBS. The transduction efficiency and CD4/CD8 ratio were determined by labeling CAR T cells with a FITC-labeled human CD19 protein, Fc Tag (CD9-HF251, ARCO, Biosystems, Beijing, China), an APC-conjugated antibody against CD8 (catalog no. 344722, BioLegend, California, USA), and a PE-Cy7-conjugated antibody against CD 4 (25-0047-42, eBioscience, San Diego, CA) at $4^{\circ} \mathrm{C}$ for $45 \mathrm{~min}$ in the dark.

For assessing the CAR T cell phenotype to analyze CAR T cell differentiation stages, CAR T cells were incubated with the following antibodies: APC-Cy7-conjugated anti-CD8 (344714, BioLegend), AF700conjugated anti-CD4 (56004942, eBioscience), PE-conjugated anti-CCR7 (353204, BioLegend), PerCPCy5.5-conjugated anti-CD45RA (304122, BioLegend), and PE-Cy7-conjugated anti-CD127 (25-1287-42, eBioscience).

CD19 expression on Raji cells was detected by staining with an APC-conjugated antibody against CD19 (302212, BioLegend), and monocyte CD14 expression was determined by staining with an Alexa Fluor 700-conjugated antibody against CD14 (325614, BioLegend).

After antibody labeling, samples were washed twice in $0.1 \mathrm{~mL}$ of PBS containing $2 \%$ FBS before detection using an Attune NxT flow cytometer (Thermo Scientific, USA). Data were analyzed using FlowJo V10 (TreeStar, USA).

\section{Cell proliferation assay}

The cell proliferation assay was performed using a carboxyfluorescein diacetate succinimidyl ester (CFSE) assay kit (Abcam, UK) following the manufacturer's instructions. In brief, $2 \times 10^{5}$ CART-19 cells were labeled with $2.5 \mathrm{mM}$ CFSE and cocultured with Raji cells, which were inactivated by exposure to 10 $\mu \mathrm{g} / \mathrm{mL}$ mitomycin C (Selleck, Houston, USA) at a ratio of 2:1 in 24-well plates in $200 \mu \mathrm{L}$ of serum-free AIM-V medium (Gibco, USA) for 5 days. CFSE was detected by flow cytometry on days 0 and 5 to analyze CAR T cell proliferation.

\section{Degranulation assay}

CD107a expression on CD8 T cells was detected by flow cytometry. In brief, $1 \times 10^{6}$ CAR T cells were cocultured with target cells at a 5:1 ratio in 96-well plates with a total of $200 \mu \mathrm{l}$ of T cell culture medium 
AIM-V (Gibco) per well, The Golgi inhibitor Monessen (Invitrogen, Carlsbad, US) and CD107a- AF700 antibody (BD, New Jersey, USA) were added before coincubation. A protease inhibitor cocktail (Invitrogen, Carlsbad, US) was added to the well containing the positive control. After $6 \mathrm{~h}$ incubation, the cells were collected and washed twice before analyzed by flow cytometry.

\section{Cytotoxicity assay}

Raji cells $\left(1 \times 10^{4}\right)$ and CART-19 cells were seeded in $200 \mu \mathrm{L}$ of serum-free RPMI 1640 medium (Gibco) at an effector : target (E:T) ratio of 10:1, 5:1, 2.5:1, or 1.25:1 in 96-well plates for $16 \mathrm{~h}$. Lactate dehydrogenase (LDH) activity in the medium was measured using a cytotoxicity detection kit (Promega, Wisconsin, USA) according to the manufacturer's protocol, and the absorption at $490 \mathrm{~nm}$ was measured using a full wavelength reader Multiskan GO (Thermo Scientific). The percentage of tumor lysis was calculated as follows: \% tumor lysis = (experimental value - low control of CART-19 cells - low control of target cells) $\times 100 /$ (high control of target cells - low control of target cells), where the low control was the assay medium + cells and the high control was the assay medium $+2 \%$ Triton $X-100+$ cells.

\section{Quantitation of cytokine levels}

Cytokine levels in culture supernatants were measured using the Th1/Th2 Cytometric Bead Array Kit II (BD Bioscience) according to manufacturer protocols. In brief, supernatants collected from the coculture system or mouse serum was incubated with fluorophore-labeled antibodies against IL-2, IL-4, IL-6, IL-10, IFN- $y$, TNF-a and IL-17A for $3 \mathrm{~h}$. After the incubation, the samples were washed with the wash solution and analyzed by flow cytometry.

\section{Quantitative RT-PCR}

Total mRNA was extracted from cells using TRIzol (Invitrogen) and converted into cDNA using HiScript ${ }^{\circledR}$ II Q RT SuperMix (Vazyme Biotech, Nanjing, China). Quantitative RT-PCR was performed using an ABI7500 system (Life Technologies, USA) and primers against IL-6 (forward: 5'-

TAACCACCCCTGACCCAACCA-3'; reverse: 5'-GCGCAGAATGAGATGAGTTGTCA-3') and a fluorescent probe (5'-AAATGCCAGCCTGCTGACGAAGCTGCA-3'). The $\mathrm{Ct}$ for samples was normalized to that of the $\beta$-actin housekeeping gene as follows: $\triangle \mathrm{Ct}$ (sample) $=\mathrm{Ct}(\mathrm{IL}-6)-\mathrm{Ct}$ ( $\beta$-actin). Expression relative to that of the control sample was calculated as follows: $2-\Delta \Delta \mathrm{Ct}=2^{\wedge}-(\Delta \mathrm{Ct}$ [sample] $-\Delta \mathrm{Ct}$ [control]). 
Total RNA was extracted using TRIZOL Reagent (Cat\#15596-018, Life technologies, Carlsbad, CA, US) following the manufacturer's instructions and checked for a RIN number to inspect RNA integrity by an Agilent Bioanalyzer 2100 (Agilent technologies, Santa Clara, CA, US). Qualified total RNA was further purified by RNAClean XP Kit (Cat A63987, Beckman Coulter, Inc. Kraemer Boulevard Brea, CA, USA) and RNase-Free DNase Set (Cat\#79254, QIAGEN, GmBH, Germany).

\section{Xenograft model}

All experiments were approved by the Institutional Animal Care and Use Committee of East China Normal University. Male NOD/SCID/Yc-/- (NSG) mice aged 4 to 6 weeks were obtained from Biocytogen (Beijing, China). Mice were injected with $1 \times 10^{6}$ Raji cells that stably expressed luciferase (Raji-luc) via the tail vein to establish a xenograft leukemia tumor model. The mice were then randomly divided into four groups and treated with saline $\left(n=3\right.$, control), $6 \times 10^{6}$ untransduced T cells $\left(n=3\right.$, negative treatment control), $6 \times 10^{6}$ regular CART-19 cells $(n=3)$, or $6 \times 10^{6}$ ssCART-19 cells $(n=3)$ on day 4. Tumor progression was monitored by bioluminescence imaging (BLI) using a Xenogeny-IVIS imaging system (Perkin Elmer, MA, USA) every 3 days beginning on day 1 . The mice were sacrificed by vertebral dislocation when moribund or upon development of hind-limb paralysis.

For in vivo imaging of Raji-luc cells, mice were injected intraperitoneally with D-luciferin (YEASEN, Shanghai, China), anesthetized with isoflurane and imaged using the Xenogeny-IVIS imaging system (Perkin Elmer).

\section{Statistical analysis}

Statistical analyses were carried out using Prism 8.0 (GraphPad Software Inc., San Diego, CA, USA). Each experiment was repeated with three biological replicates, and data are presented as the mean $\pm S D$. The group means from degranulation assays and cytokine secretion assays were compared using one-way analysis of variance (ANOVA). Differences among treatment groups and control groups in vitro killing assays were analyzed using two-way ANOVA followed by Dunnett's multiple-comparison test. Differences associated with a $p$-value $<0.05$ were considered significant.

\section{Results}

\section{IL-6 derived from activated regular CART-19 cells triggers monocyte release of IL-6}

To determine the effect of cytokines from activated regular CART-19 cells on monocytes, we first examined whether regular CART-19 cells increase the expression of IL-6 and other cytokines upon 
encountering CD19 + cells. We cocultured regular CART-19 cells with CD19-expressing Burkitt human B lymphoma (Raji) cells in an "effector/target" coculture model system. CD19 expression on Raji cells was confirmed by FACS (Additional file 1: Figure S1a). Significant increases in the amounts of both IL-6 mRNA production and IL- 6 cytokine production were observed in the cocultured regular CART-19 cells compared with nonactivated regular CART-19 cells, with the increases being 4-fold and 20-fold higher, respectively (Fig. 1A and B), which demonstrated that regular CART-19 cells could significantly increase IL-6 mRNA and protein expression once induced specifically by Raji cells.

We next examined whether the enhanced IL- 6 production by activated regular CART-19 cells triggers monocytes to increase the release of IL- 6 and other cytokines by adding supernatants from CAR T/Raji cell cocultures to CD14-expressing monocytes (see Additional file 1: Figure S1b for confirmation of CD14 expression). Significantly higher IL-6 levels in the monocyte culture medium were observed after $24 \mathrm{~h}$ of incubation with the supernatant of the "effector/target" coculture than immediately after beginning the incubation, which was used as the baseline level for comparison (Fig. 1C). In contrast, there were no differences in IL-6 or other cytokine levels in the monocyte culture medium after the addition of supernatants from untransduced T cells cocultured with Raji cells when comparing the same study time points. To pinpoint which cytokines are the main triggering factors that enhance IL- 6 release from monocytes, we incubated primary monocytes with recombinant IL-1, IL-2, IL-6, or IFN-y for 48 h, and then the released IL- 6 level was measured by flow cytometry. To determine whether the enhancement in the IL6 level is derived from activated monocytes, the amount of exogenous IL- 6 was subtracted from the level measured in the monocyte culture medium. Incubation with IL-6 triggered a remarkable enhancement in the release of IL- 6 from monocytes, with almost 100 times more IL- 6 release in the IL-6-treated group than in the negative control group that was not incubated with any cytokines (Fig. 1D). The increases in IL-6 levels were also observed to be 25 and 3 times higher for the monocytes incubated with IL-1 or IFN-y than for the negative control monocytes, respectively. In contrast, IL-2 did not trigger IL- 6 release. These data demonstrated that IL- 6 was the most potent cytokine in triggering monocyte IL- 6 release under our experimental conditions.

IL-6 knockdown does not impair basic properties of the SSCART-19 T cells

To investigate whether the introduction of an IL-6-specific shRNA to regular CART-19 cells changes basic cell properties, we first designed 8 different IL-6-targeting shRNA sequences and cloned them into lentiviral vectors containing the CAR construct. IL- 6 shRNA-2, which had the best IL- 6 gene knockdown efficiency (70\%) (Fig. 2A) and the highest IFN-y/IL-6 mRNA ratio (73\%) (Fig. 2B), was selected for this study. We then constructed CAR vectors containing a 4-1BB costimulatory domain, CD3 zeta domain and CD19-targeted single-chain variable fragment (FMC63) with or without IL-6 shRNA-2 (Fig. 2C). T cells transduced with CD19-CAR were called regular CART-19 cells, and those transduced with CD19-CAR and IL-6 shRNA-2 were designated SSCART-19 cells for subsequent experiments.

Then, the basic properties of ssCART-19 cells, including transduction efficiency, the CD4/CD8 ratio after transduction, proliferation, and cytotoxicity to the corresponding cancer cells were characterized and 
compared with the same features of regular CART-19 cells. The SSCART-19 cells showed cell properties similar to those of the regular CAR T-19 cells in terms of the level of transduction efficiency $(45.7 \% \mathrm{vs}$ $38.1 \%$ ), the ratio of CD4/CD8 (Fig. 2D), proliferation (Fig. 2E) and cytotoxicity as measured by a CD107a (Fig. 2F) degranulation assay or cytotoxicity assay when cocultured with either Raji cells or patientderived primary B-ALL cells (Fig. 2G). Thus, the results presented here indicated that compared with the regular CART-19 cells without the IL-6-specific shRNA modification, the SSCART-19 cells modified with the IL-6-specific shRNA did not show significant differences from basic $T$ cell functions.

IL-6 knockdown in ssCART-19 cells reduces IL-6 release from monocytes

To investigate whether monocyte release of IL- 6 can be reduced by knocking down IL- 6 expression in sSCART-19 cells, we first confirmed that shRNA-mediated IL- 6 knockdown in sSCART-19 cells could significantly reduce soluble IL-6 protein levels to 4 -fold lower than the levels in regular CART-19 cells (Fig. 3A). Next, we found that IL-6 mRNA levels were reduced by up to 70\% in ssCART-19 cells compared to regular CART-19 cells (Fig. 3B).

We investigated the effect of IL- 6 knockdown in SSCART-19 cells on monocyte IL- 6 secretion by adding the supernatant from a coculture of ssCART-19 cells and Raji cells to monocytes. Significantly lower IL-6 levels were observed in the monocyte supernatant after the addition of the supernatant from SSCART19/Raji cell cocultures than after the addition of the supernatant from regular CART-19/Raji cell cocultures (Fig. 3C). These results indicated that IL-6 knockdown in sSCART-19 cells could remarkably reduce IL-6 secretion by monocytes.

To confirm that IL-6-specific shRNA knockdown activity is stable, specific and continuous, we repeatedly stimulated ssCART-19 cells with Raji cells every $36 \mathrm{~h}$ and continuously monitored IL- 6 levels in the supernatant. The sSCART-19 cells consistently maintained lower IL- 6 levels than regular CART-19 cells over 6 days, regardless of whether they were re-stimulated with Raji cells (Fig. 3D), while the IL-6 expression level of the regular CART-19 cells was significantly enhanced upon re-stimulation with Raji cells. Thus, IL-6-specific shRNA-mediated knockdown could be continuously maintained in ssCART-19 cells without being affected by repeated stimulation with target cells during the experimental period.

IL-6 knockdown in sSCART-19 cells does not show significant off-target effects

To determine whether IL- 6 knockdown affects SsCART-19 cell function, we measured IFN- $y$ and IL-2 release in sSCART-19 cells. Without Raji cell stimulation, the levels of IFN- $\gamma$ and IL-2 release from both regular CART-19 and SSCART-19 cells were maintained at baseline levels for the 6 days of the experiment. When activated with Raji cells, the increases in the levels of IFN- $\gamma$ (Fig. 3E) and IL-2 (Fig. 3F) for the sSCART-19 and regular CART-19 cells were observed to have similar enhancement profiles, which indicated that IL-6 knockdown did not affect IFN- $\gamma$ or IL-2 expression in CART-19 cells.

We also investigated whether IL- 6 knockdown affects the differentiation of sSCART-19 cells by measuring the Tscm, Tcm, Tem and Teff ratios (indicative of different differentiation stages) of populations of CD8 
+ SSCART-19 cells in comparison to those of population of regular CART-19 cells derived from the same healthy donor. Similar differentiation profiles were observed in both CD8 + T cells for SSCART-19 and regular CART-19 cells (Additional File S2A), which indicated that the modification with the IL-6-specific shRNA knockdown system did not affect the differentiation of sSCART-19 cells.

To determine whether IL- 6 knockdown affects the expression of other genes in cells, RNA sequencing (RNA-seq) analysis was conducted. The mRNAs of both regular CART-19 cells and ssCART-19 cells were analyzed by RNA-sEq. Compared with the gene expression profiles of both ssCART-19 and regular CART19 cells, those of sSCART-19 cells showed altered Jak-STAT signaling pathway (CISH), which is involved in IL-6 production (Additional File S2B). Additionally, gene ontology enrichment analysis showed that IL-6 knockdown did not affect T cell activation, cell proliferation or cell differentiation (Additional File S2C), consistent with the results of our in vitro assays. The RNA-seq data indicated that IL-6-specific shRNA knockdown did not obviously produce "off-target effects" that were disease related or T cell function related.

IL-6 knockdown in SSCART-19 cells improves in vivo safety and preserves antitumor efficacy

We next assessed the antileukemic activity of SSCART-19 cells in vivo using a xenograft mouse model (see schematic in Fig. 4A) established by injecting NSG mice with $1 \times 10^{6}$ Raji-luc cells on day 0; mice were treated by intravenous infusion of equal numbers of SSCART-19 or regular CART-19 cells $\left(6 \times 10^{6}\right) 4$ days later. Tumor progression was monitored by BLI every 3 days beginning on day 1 . Control mice in the negative control groups were treated with untransduced (NC) T cells or freezing medium and showed rapid progression of leukemia. In contrast, the mice treated with either regular CART-19 or SSCART-19 cells showed obvious inhibition of tumor progression compared to the control mice (Fig. 4B) and increased survival rates (Fig. 4C), and there was no significant difference between the SSCART-19 and regular CART19 cells, which indicated that SSCART-19 cells had therapeutic efficacies equivalent to those of regular CART-19 cells. As expected, IL-6 levels were significantly lower in the serum of the ssCART-19 cell-treated mice than in that of the regular CART-19 cell-treated mice (Fig. 5A). Importantly, the serum IFN-y levels in the SSCART-19 cell-treated mice were comparable to those in the regular CART-19 cell-treated mice (Fig. 5B). The mice treated with SSCART-19 cells showed slightly slower but comparable expansion abilities in both total $T$ cells and CAR $+T$ cells than those treated with regular CART-19 cells ( Fig. 5C\&5D ). Our results demonstrated that compared to regular CART-19 cells, SSCART-19 cells could significantly reduce the IL- 6 level in treated mice, but importantly, SSCART-19 cells also showed remarkable antitumor efficacy with IFN- $\gamma$ release profiles similar to those of regular CART-19 cells under the experimental conditions.

\section{Discussion}

Although CAR T cell therapy has shown remarkable success in the treatment of refractory and relapsed hematological malignancies, safety is a major concern in the clinic due to the life-threatening side effects of severe cytokine release syndrome (sCRS) and severe CAR-T-related encephalopathy (sCRES) [24, 25]. 
Both sCRS and sCRES are commonly considered to be caused by rapidly elevated levels of proinflammatory cytokines, especially IL-6, after CAR T cell activation by the corresponding target expression on target cells[2]. These proinflammatory cytokines are reportedly expressed by many immune cells, including T cells, B cells, monocytes (dendritic cells and macrophages), and endothelial cells[12, 15].

In this study, we showed that IL- 6 secreted by activated regular CART-19 cells triggered the release of relatively large amounts of IL- 6 and other cytokines from monocytes. This cascade may be the primary mechanism underlying CRS during CAR T cell therapy. We modified CAR T cells with an IL-6-specific shRNA system to reduce their IL- 6 expression. An in vitro study demonstrated that IL- 6 knockdown by the IL-6-specific shRNA in ssCART-19 cells significantly reduced ssCART-19 cells IL-6 expression, which in turn reduced the stimulatory activity that induced monocytes to release IL-6. Moreover, the IL- 6 knockdown in SSCART-19 cells was maintained during repeated stimulation with B lymphoma cells for 6 days in the study setting, indicating that the IL-6-specific shRNA could act as a long-term suppressor of IL-6 in CAR T cell therapy.

On the other hand, IL-1 has been reported to be a main proinflammatory cytokine in inducing monocyte IL-6 release[6]. In our in vitro study, the addition of IL-6 produced more potent induction of monocyte release of IL- 6 and proinflammatory cytokines than did IL-1, which suggests that IL- 6 secreted by CAR T cells is one of the key initiator for triggering monocyte IL- 6 release and promoting CRS development in patients treated with CAR T cell therapy.

Previous studies have shown that blocking IL-6/IL-6R does not affect the properties or therapeutic efficacy of CAR T cells[21]. Consistent with these observations, we found that SSCART-19 cells exhibited cell functions and anticancer activities similar to those of regular CART-19 cells in terms of response to tumor antigen stimulation, cell proliferation, and cytotoxicity to CD19 + cell lines and patient-derived primary malignant B cells. These data suggest that IL-6 knockdown in SSCART-19 cells may reduce the risk of CRS and CRES without sacrificing antitumor efficacy.

Last, we demonstrated that serum IL-6 levels were lower in a mouse xenograft model of leukemia after treatment with ssCART-19 cells than after treatment with regular CART-19 cells. The two types of CAR T cells showed similar antitumor toxicity, strengthening the hypothesis that IL- 6 knockdown can improve the safety of ssCART-19 cell treatments without reducing antitumor effects in vivo.

The findings of our study are similar to those of a recent study that reported that GM-CSF knockout using CRISPR/Cas 9 could reduce the sCRS rate and enhance T cell function in mice[25]. The shRNA-mediated gene knockdown, which silences endogenous genes at the RNA level, is one of the most common therapeutic approaches to treat genetic and acquired diseases[26]. One potential advantage of the shRNA gene knockdown technology approach compared to CRISPR/Cas9 gene knockout technology is that it avoids "off-target" genome editing effects at the DNA level that may cause unpredictable severe side effects, and complete abolishment of endogenous GM-CSF gene expression may also cause CAR T cells to fail to respond to physiological homeostasis needs. Given the incomplete understanding of the roles of 
each cytokine in the complicated human immune system and the potential "off-target" risks of CRISPR/Cas9 gene editing technology itself, the shRNA knockdown approach may be safer[27]. IL-6 knockdown also did not compromise $\mathrm{T}$ cell function, proliferation, differentiation or cytotoxicity.

In this study, we systematically evaluated the effects of IL- 6 knockdown in SSCART-19 cells both in vitro and in vivo as a potential strategy to reduce the side effects of CAR T cell treatment. By knocking down IL- 6 expression in SSCART-19 cells, the strength of the stimulation that triggers monocyte IL- 6 release activity was reduced, which may further reduce the incidence of SCRS and improve safety profiles in the clinic. This approach may have major potential benefits for increasing patient survival, reducing the need for costly tocilizumab treatment, and decreasing the risk of neurological complications caused by CAR T cell therapy.

\section{Conclusion}

The rapid elevation in CART-19-derived IL- 6 expression induced by CD19-expressing cells may be one of the major triggering factors that enhances monocyte release of IL-6 and other proinflammatory cytokines, which may drive CRS development. Reducing the ssCART-19 cell-derived IL-6 expression level with an IL-6specific shRNA could significantly reduce IL- 6 release by monocytes, which could potentially decrease the severity or incidence of SCRS and related complications, without reducing SSCART-19 cell antitumor efficacy in vitro and in vivo. Thus, IL-6 knockdown in ssCART-19 cells may be a promising approach for improving safety in treating refractory and relapsed $B$ lymphocyte leukemia and may also be used in other CAR T cells for improving clinical safety when treating other malignancies.

\section{Abbreviations}

B-ALL

B cell acute lymphoblastic leukemia;

CAR

chimeric antigen receptor;

CFSE

carboxyfluorescein diacetate succinimidyl ester;

GM-CSF

granulocyte macrophage colony-stimulating factor;

IFNy

interferon-ץ;

IL-6

interleukin-6;

IL-1

interleukin-1;

IL-2

interleukin-2; 
NSG

NOD/SCID IL-2RyCnull;

$\mathrm{NC}$

untransduced;

PBMCs

peripheral blood mononuclear cells;

PBS

phosphate-buffered saline;

scFv

single-chain variable fragment;

TNF-a

tumor necrosis factor-a.

\section{Declarations}

Ethics approval and consent to participate

Not applicable

\section{Protection of Animal subjects}

The institutional and national guidelines for the care and use of laboratory animals were followed and overseen by the animal facility of the East China Normal University.

\section{Consent for publication}

Not applicable

\section{Availability of data and material}

The data-sets used and/or analyzed during the current study are available from the corresponding author on reasonable request.

\section{Competing interests}

The authors declare no conflict of interest.

\section{Funding}

This study is supported by grants from Shanghai Engineering Research Center of Molecular Therapeutics and New Drug Development, School of Chemistry and Molecular Engineering, East China Normal University; Shanghai Unicar-Therapy Bio-medicine Technology Co.,Ltd and Institute of Biomedical Engineering and Technology; Frontier Clinical Technical Project of the Science and Technology Department of Jiangsu Province (BE2017655). 


\section{Authors' contributions}

LY, DPW, LQK and XWT contributed to the concept development and study design and wrote the manuscript. NX and MHL participated in the figure preparation and revised the paper. All authors read and approved the final manuscript.

\section{Acknowledgement}

We thank Mira Peled-Kamar for revising the manuscript.

\section{Publisher's Note}

Springer Nature remains neutral with regard to jurisdictional claims in published maps and institutional affiliations.

\section{References}

[1] Sadelain M, Rivière I and Riddell SJN. Therapeutic T cell engineering. Nature. 2017;545: 423-431

[2] Gust J, Hay KA, Hanafi L-A, et al. Endothelial activation and blood-brain barrier disruption in neurotoxicity after adoptive immunotherapy with CD19 CAR-T cells. Cancer Discov. 2017;7: 1404-1419

[3] Trinschek B, Lüssi F, Haas J, et al. Kinetics of IL-6 production defines T effector cell responsiveness to regulatory T cells in multiple sclerosis. PLoS One. 2013;8: e77634

[4] Brudno JN and Kochenderfer JNJB, The Journal of the American Society of Hematology. Toxicities of chimeric antigen receptor T cells: recognition and management. Blood. 2016;127: 3321-3330

[5] Santomasso BD, Park JH, Salloum D, et al. Clinical and biological correlates of neurotoxicity associated with CAR T-cell therapy in patients with B-cell acute lymphoblastic leukemia. Cancer Discov. 2018;8: 958-971

[6] Wang X, Popplewell LL, Wagner JR, et al. Phase 1 studies of central memory-derived CD19 CAR Tcell therapy following autologous HSCT in patients with B-cell NHL. Blood. 2016;127: 2980-2990

[7] Porter D, Frey N, Wood PA, et al. Grading of cytokine release syndrome associated with the CAR T cell therapy tisagenlecleucel. J Hematol Oncol. 2018;11: 35.

[8] Brudno JN and Kochenderfer JNJBr. Recent advances in CAR T-cell toxicity: mechanisms, manifestations and management. Blood Rev. 2019;34: 45-55 
[9] Maus MV, Haas AR, Beatty GL, et al. T cells expressing chimeric antigen receptors can cause anaphylaxis in humans. Cancer Immunol Res. 2013;1: 26-31

[10] Brentjens RJ, Davila ML, Riviere I, et al. CD19-targeted T cells rapidly induce molecular remissions in adults with chemotherapy-refractory acute lymphoblastic leukemia. Sci TransI Med.2013;5: 177ra138

[11] Davila ML, Riviere I, Wang X, et al. Efficacy and toxicity management of 19-28z CAR T cell therapy in B cell acute lymphoblastic leukemia. Sci Transl Med. 2014;6: 224ra225

[12] Hay KA, Hanafi L-A, Li D, et al. Kinetics and biomarkers of severe cytokine release syndrome after CD19 chimeric antigen receptor-modified T-cell therapy. Blood. 2017;130: 2295-2306

[13] Turtle CJ, Hanafi L-A, Berger C, et al. CD19 CAR-T cells of defined CD4+: CD8+ composition in adult B cell ALL patients. J Clin Invest. 2016;126: 2123-2138

[14] Locke FL, Ghobadi A, Jacobson CA, et al. Long-term safety and activity of axicabtagene ciloleucel in refractory large B-cell lymphoma (ZUMA-1): a single-arm, multicentre, phase 1-2 trial. Lancet Oncol. 2019;20: 31-42

[15] Neelapu SS, Locke FL, Bartlett NL, et al. Axicabtagene ciloleucel CAR T-cell therapy in refractory large B-cell lymphoma. N Engl J Med. 2017;377: 2531-2544

[16] Giavridis T, van der Stegen SJ, Eyquem J, et al. CAR T cell-induced cytokine release syndrome is mediated by macrophages and abated by IL-1 blockade. Nat Med. 2018;24: 731-738

[17] Gofshteyn JS, Shaw PA, Teachey DT, et al. Neurotoxicity after CTL019 in a pediatric and young adult cohort. Ann Neurol. 2018;84: 537-546

[18] Leech MD, Barr TA, Turner DG, et al. Cutting Edge: IL-6-Dependent Autoimmune Disease: Dendritic Cells as a Sufficient, but Transient, Source. J Immunol. 2013;190: 881-885

[19] Barr TA, Shen P, Brown S, et al. B cell depletion therapy ameliorates autoimmune disease through ablation of IL-6-producing B cells. J Exp Med. 2012;209: 1001-1010

[20] Schulert GS and Grom AAJArom. Pathogenesis of macrophage activation syndrome and potential for cytokine-directed therapies. Annu Rev Med. 2015;66: 145-159

[21] Singh N, Hofmann TJ, Gershenson Z, et al. Monocyte lineage-derived IL-6 does not affect chimeric antigen receptor T-cell function. Cytotherapy. 2017;19: 867-880

[22] Mackall CL and Miklos DBJCd. CNS endothelial cell activation emerges as a driver of CAR T cellassociated neurotoxicity. Cancer Discov. 2017;7: 1371-1373

[23] Han TH, Jin P, Ren J, et al. Evaluation of three clinical dendritic cell maturation protocols containing lipopolysaccharide and interferon-gamma. J Immunother. 2009;32: 399. 
[24] Rubin DB, Danish HH, Ali AB, et al. Neurological toxicities associated with chimeric antigen receptor T-cell therapy. Brain. 2019;142: 1334-1348

[25] Sterner RM, Sakemura R, Cox MJ, et al. GM-CSF inhibition reduces cytokine release syndrome and neuroinflammation but enhances CAR-T cell function in xenografts. Blood. 2019;133: 697-709

[26] Ramishetti S and Peer DJAddr. Engineering lymphocytes with RNAi. Adv Drug Deliv Rev. 2019;141: $55-66$

[27] Cradick TJ, Fine EJ, Antico CJ, et al. CRISPR/Cas9 systems targeting $\beta$-globin and CCR5 genes have substantial off-target activity. Nucleic Acids Res. 2013;41: 9584-9592

Figures
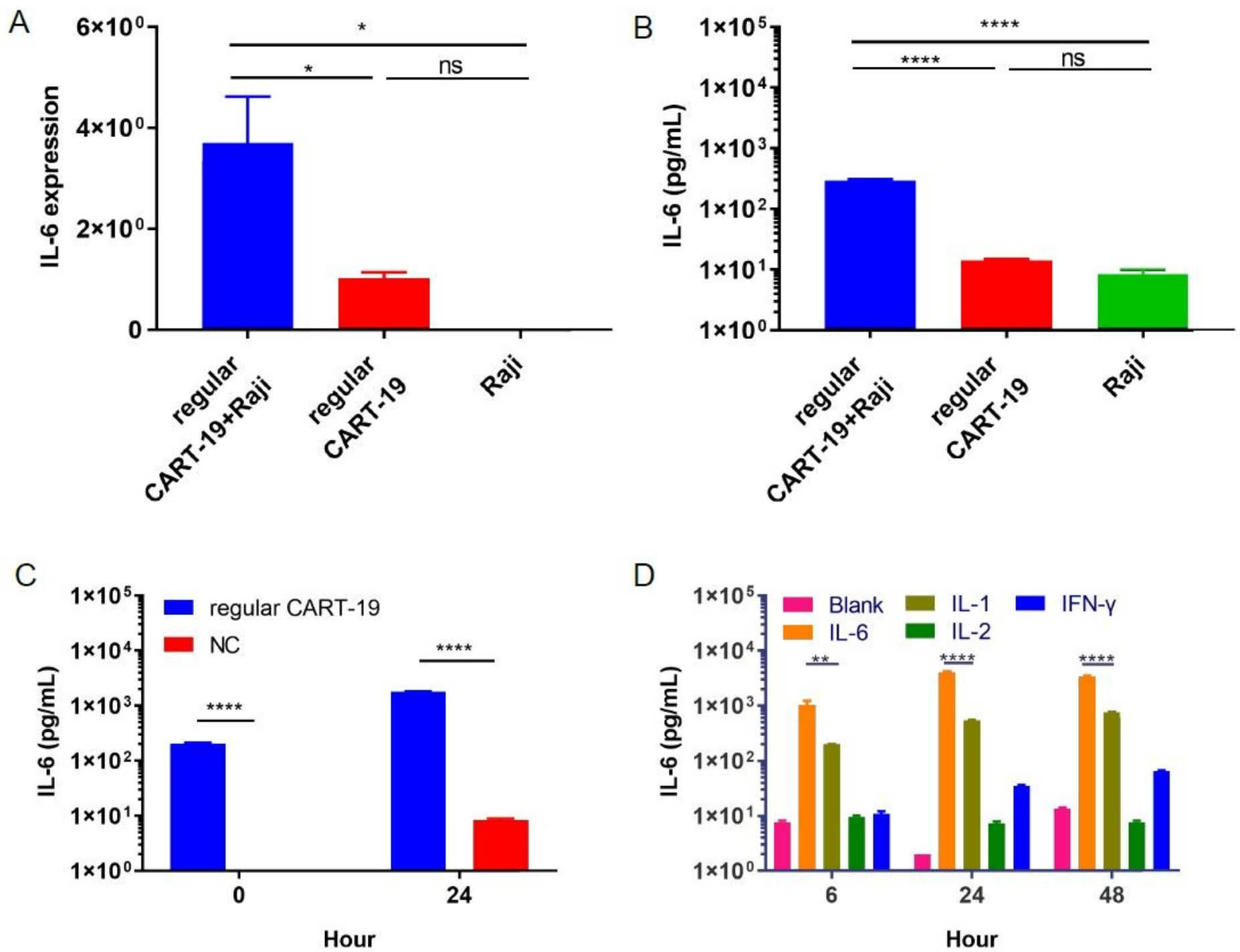

Figure 2 
IL-6 derived from activated CART-19 cells triggers monocyte release of IL-6. A) IL-6 mRNA levels in CART19 cells measured by quantitative PCR. Regular CART-19 cocultured with Raji cells for 24 h, IL- 6 mRNA levels were measured by quantitative PCR. Data are shown as the mean $\pm S D(n=3)$. NS = no significant difference, ${ }^{*} p<0.05$. B) IL-6 cytokines level secreted into the supernatant. IL- 6 protein levels in the supernatants of in CART-19 cells cocultured with Raji cells for $24 \mathrm{~h}$, were measured using the Th1/Th2 Cytometric Bead Array Kit II. NS, no significant difference, $* \star \star \star ~ p<0.0001$. C) IL-6 protein levels in the supernatants of cultures of monocytes incubated with supernatants from CART-19/Raji cell cocultures, as measured using the Th1/Th2 Cytometric Bead Array Kit II. NC, untransduced T cells cocultured with Raji cells. D) IL-6 protein levels in monocyte culture medium after the addition of recombinant IL-1, IL-2, IL-6 or IFN- $y$. Data are shown as the mean \pm SD $(n=3)$. Student's $t$ test was used to assess differences between groups. ${ }^{\star \star} p \leq 0.01,{ }^{\star \star \star \star} p \leq 0.0001$. 


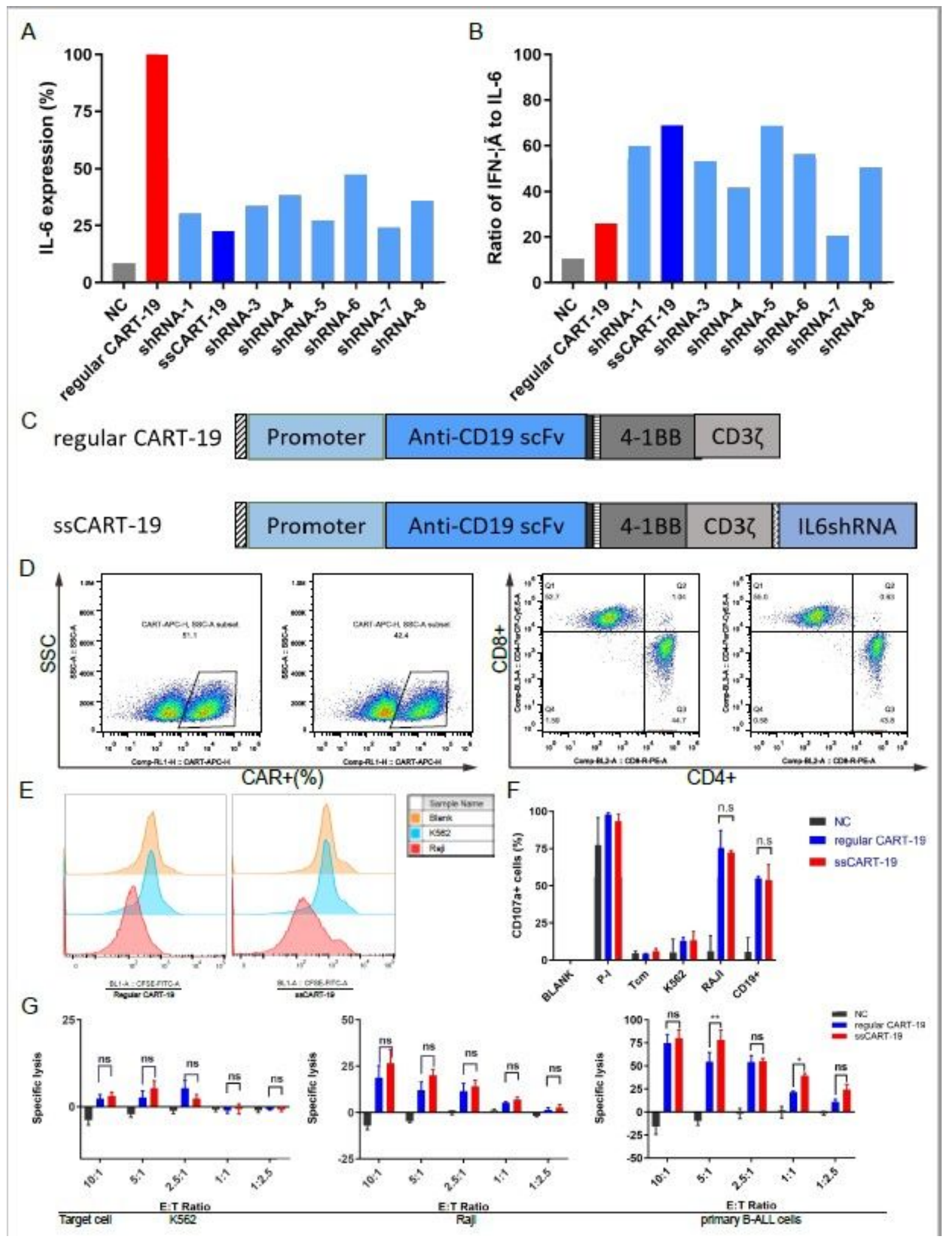

Figure 4

IL-6 knockdown in ssCART-19 T cells does not impair basic properties of CAR T cells. A) IL-6 mRNA levels and B) IFN- - /IL-6 mRNA ratios in CART-19 cells expressing 8 different IL-6-specific shRNAs. C) Schematic of the CD19 CAR vector containing an anti-human CD19 scFv linked to 4-1BB costimulatory domains and CD3- signaling domain with (sSCART-19) or without (regular CART-19) an IL-6-specific shRNA modification. D) Transduction efficiency and the CD4/CD8 ratio of ssCART-19 and CART-19 cells. E) Cell 
proliferation of sSCART-19 cells and regular CART-19 cells after re-stimulation with Raji cells, as analyzed by flow cytometry. F) CD107a expression in SSCART-19 cells and regular CART-19 cells after induction with Raji cells. G) Cytotoxicity of sSCART-19 and CART-19 cells to K562 cells, Raji cells and autologous primary acute $B$ lymphocytic leukemia cells at different effector: target ratios. Differences among groups were assessed for significance by using one-way ANOVA. Data are shown as the mean $\pm S D(n=3)$. NS, no significant difference, ${ }^{*} \mathrm{p} \leq 0.05,{ }^{* *} \mathrm{p} \leq 0.01 ;{ }^{* *} \mathrm{p} \leq 0.001,{ }^{* \star * *} \mathrm{p} \leq 0.0001$

A
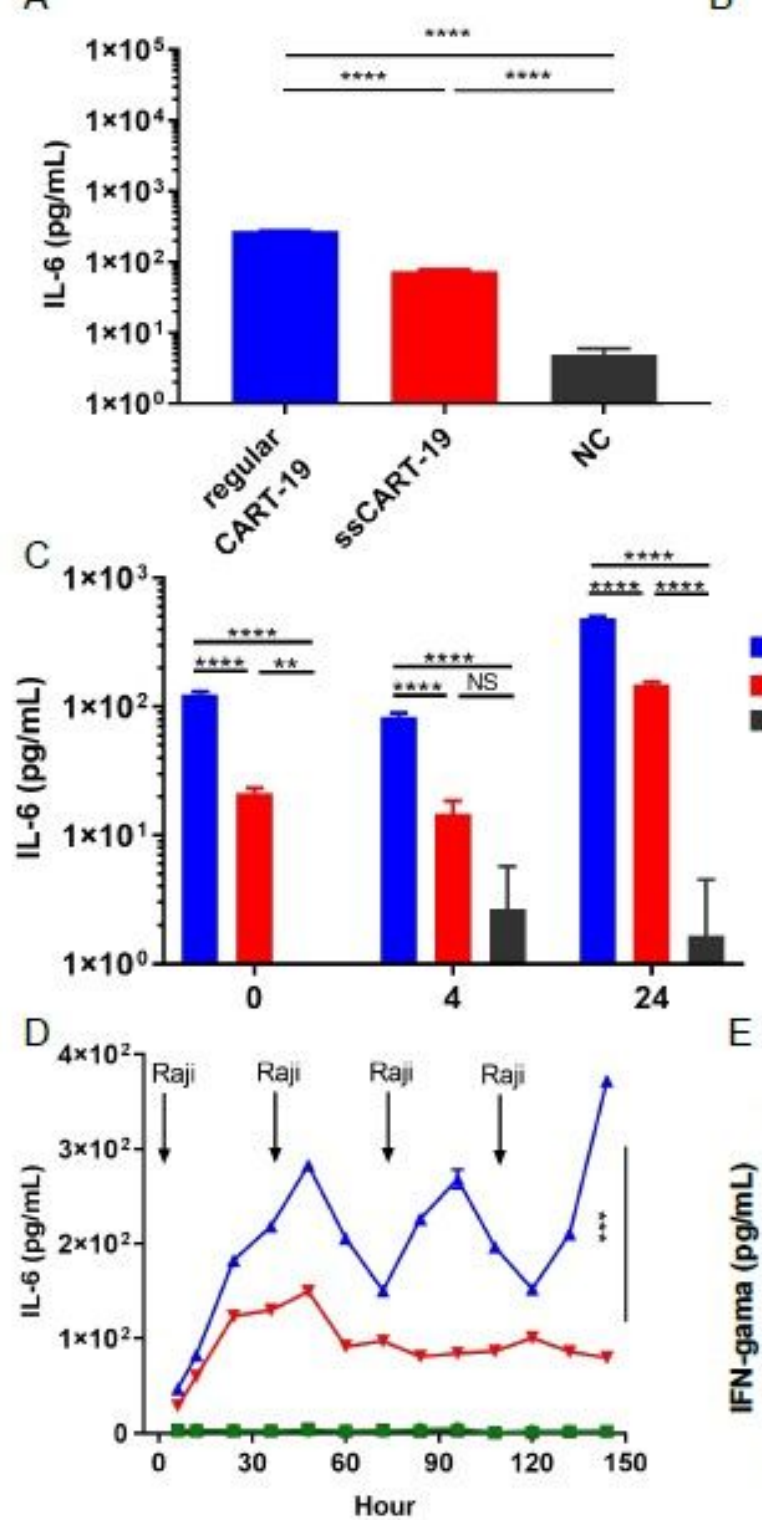

E

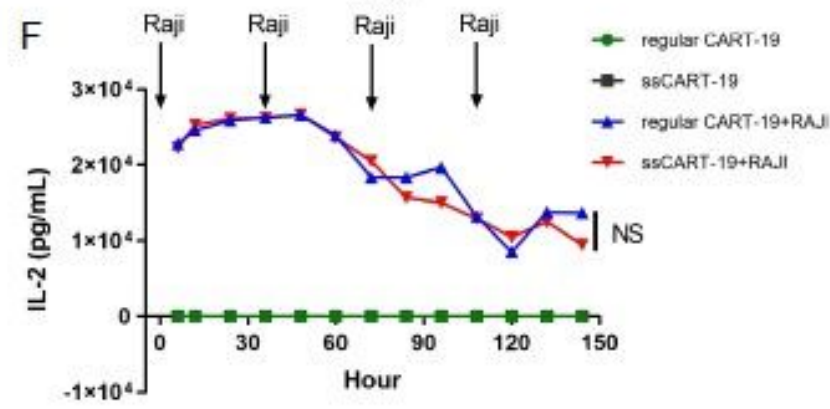

B

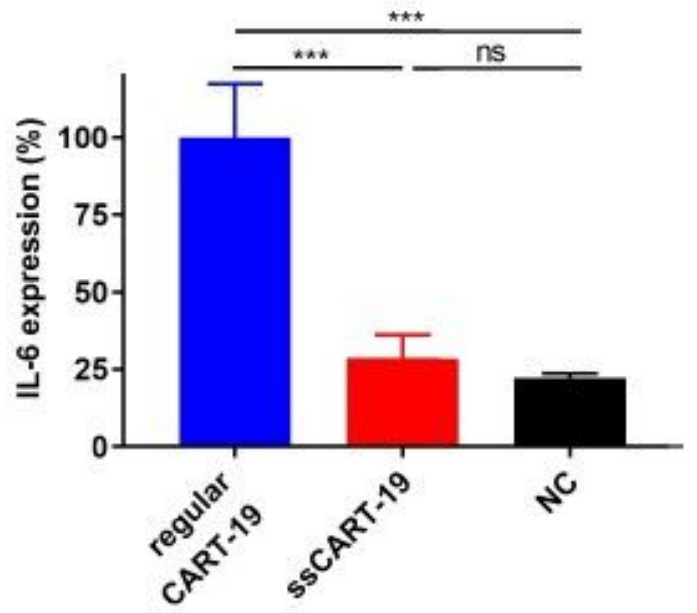

- regular CART-19

ssCART-19

- $\mathrm{NC}$

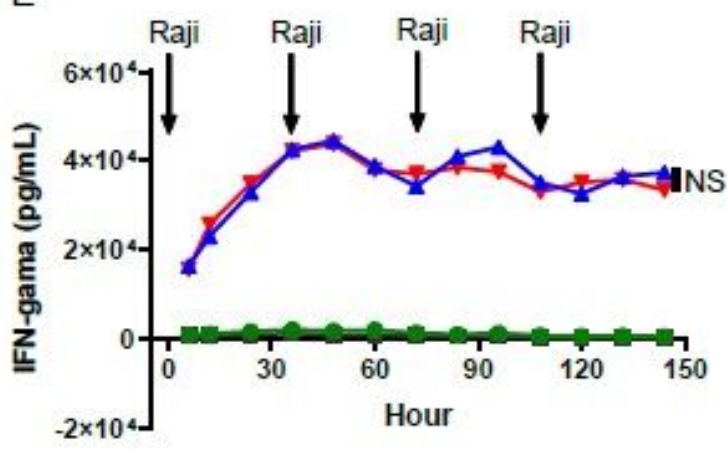

Figure 6 
IL-6 knockdown in ssCART-19 T cells reduces IL-6 release from monocytes. A) IL-6 mRNA levels and B) IL6 protein levels in sSCART-19 cells and regular CART-19 cells after stimulation with Raji. Differences were assessed for significance using one-way ANOVA. Data are expressed as the mean + SD. NS, no significant difference, ${ }^{* \star} p<0.001$. C) IL-6 levels in the medium of primary monocyte cultures incubated with supernatants from regular CART-19 or ssCART-19 cells cocultured with Raji cells. Differences were assessed using one-way ANOVA. NS, no significant difference. ${ }^{* \star} p \leq 0.01 ;{ }^{* \star} p \leq 0.001,{ }^{\star \star \star \star} p \leq 00.0001$. D) IL-6, E) IFN- $y$ and F) IL-2 protein levels in the supernatants of sSCART-19 or regular CART-19 cells cocultured with Raji cells. The cells were re-stimulated every $36 \mathrm{~h}$ as indicated by the black arrows. Differences were assessed for significance using a paired T test. ${ }^{\star \star *} \mathrm{p}<0.001$. 
A
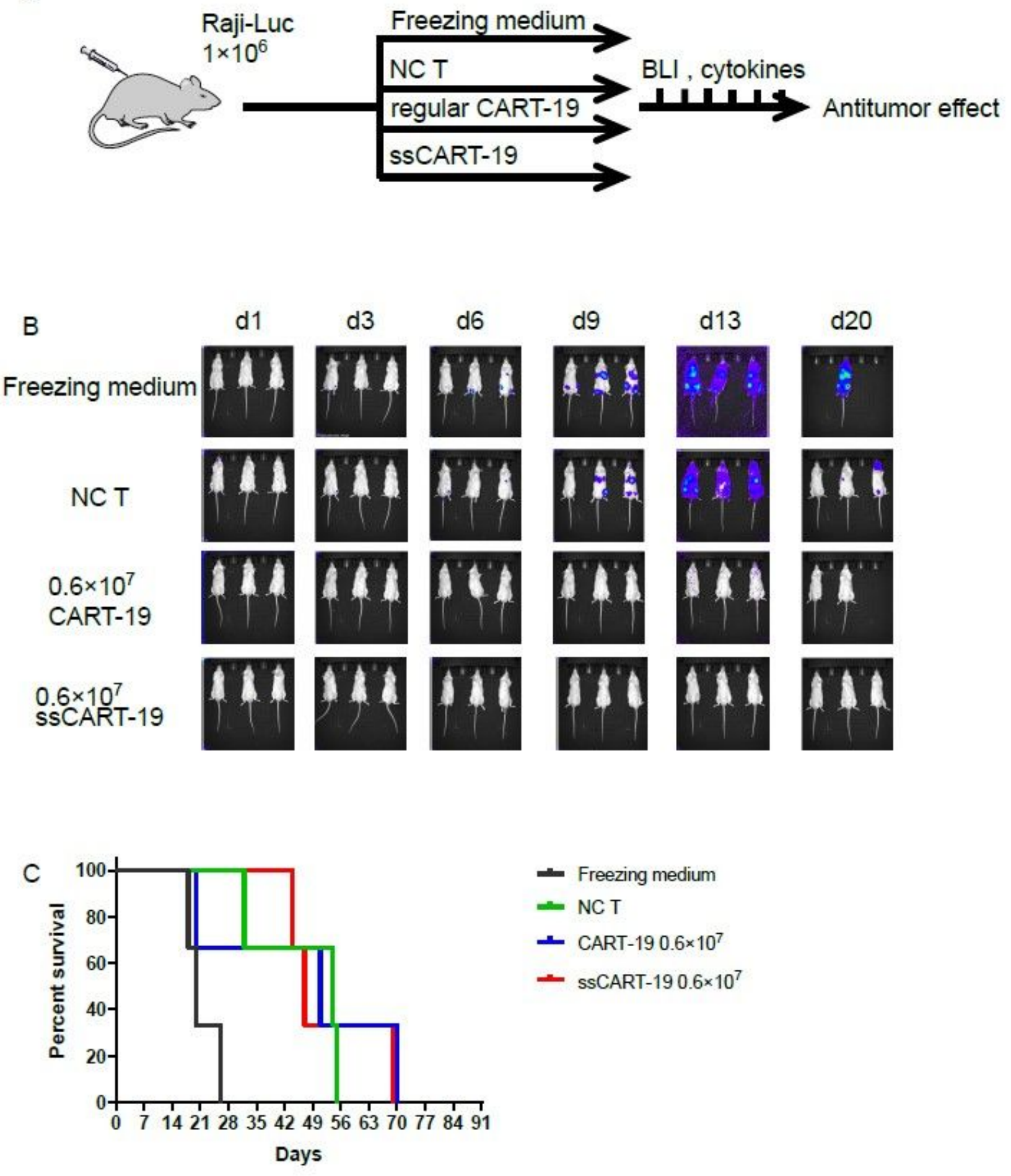

Figure 8

IL-6 knockdown in ssCART-19 cells preserves antitumor efficacy in vivo. A) Schematic of the Raji-luc xenograft model. NOD/SCID/Yc-/- (NSG) mice were injected via the tail vein with 1×106 Raji-luc cells on day 0 . Bioluminescence imaging (BLI) was performed on day 1 to quantify engraftment. The mice were then randomized and treated with freezing medium $(n=3)$, untransduced T cells $(n=3), 6 \times 106$ regular CART-19 cells $(n=3)$, or $6 \times 106$ ssCART-19 cells $(n=3)$. B) Images showing BLI of tumor growth in mice. 
Mice treated with both sSCART-19 cells and regular CART-19 cells showed obviously tumor inhibition, however, rapidly tumor progression were observed in the mice treated untransduced T cells, or freezing medium. C) Survival of mice treated with regular CART-19 cells, sSCART-19 cells, untransduced T cells, or freezing medium.
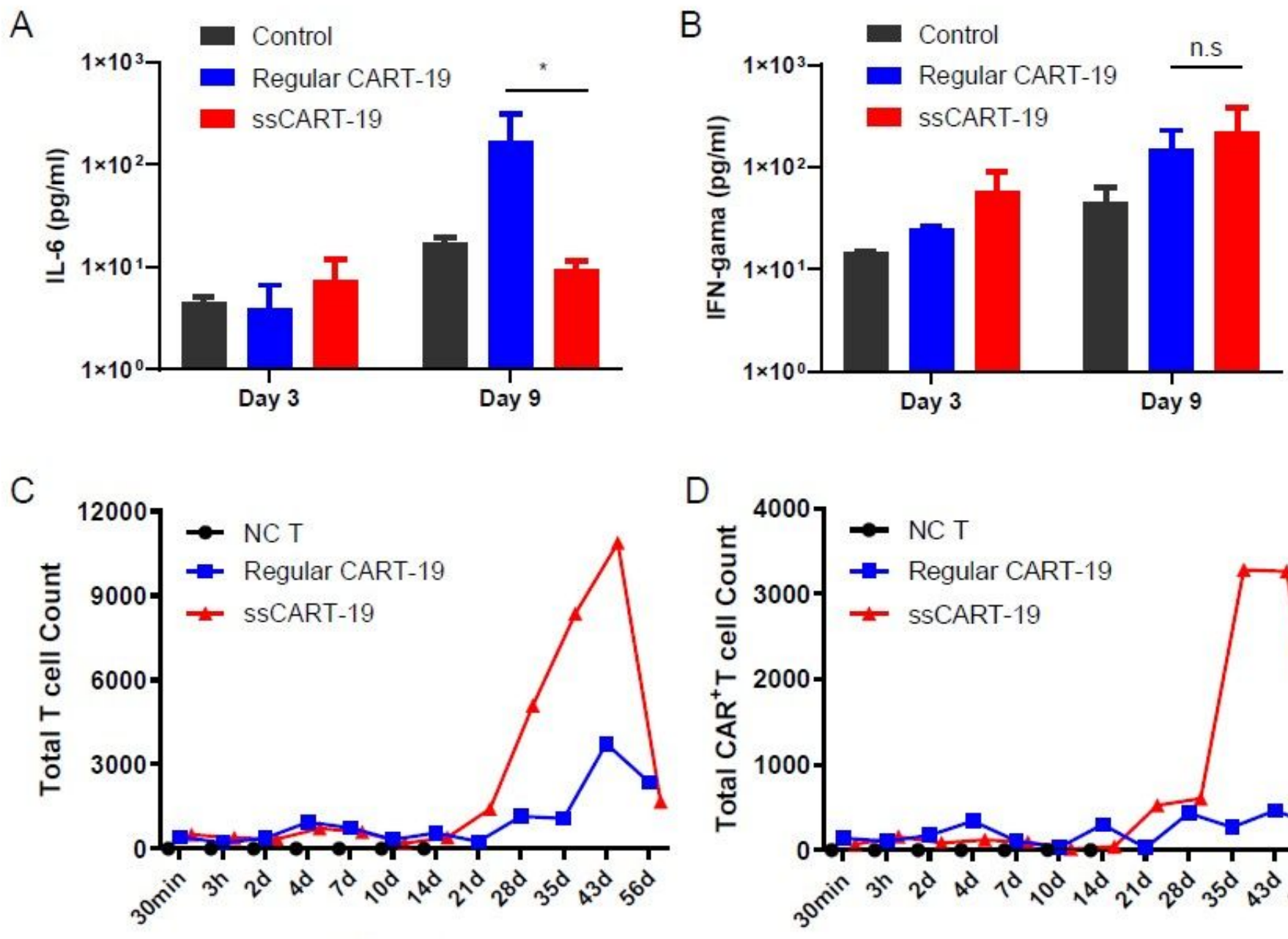

Time points

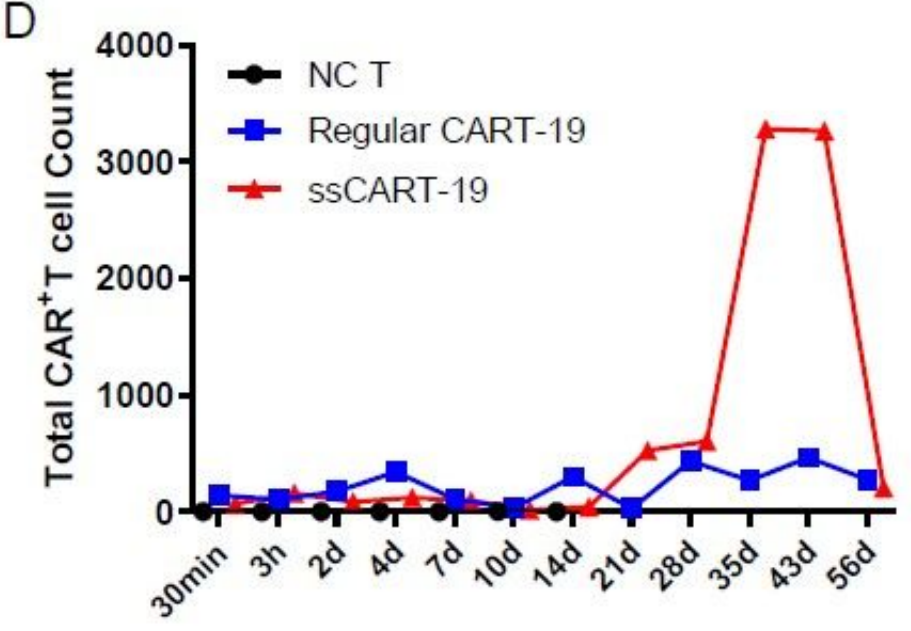

Time points

Figure 10

IL-6 knockdown in sSCART-19 cells reduce the IL-6 release in vivo. A) Serum levels of IL-6 and B) IFN- $y$ in the peripheral blood of mice treated with regular CART-19 cells or SSCART-19 cells. Blood was taken on day 3 ( 1 day before CART-19 cell administration) and day 9 ( 5 days after CART-19 cell administration). C) Total T cells count and D) Total CAR+T cells count in the peripheral blood of mice treated with regular CART-19 cells, ssCART-19 cells, untransduced T cells, or freezing medium measured by flow cytometry.

\section{Supplementary Files}

This is a list of supplementary files associated with this preprint. Click to download. 
- S2.pdf

- S2.pdf

- S1.pdf

- S1.pdf

Page 24/24 